Terapia Psicológica, vol. 39, no. 2 (julio 202I)

\begin{abstract}
1
$10.4067 /$ S0718-48082021000200237

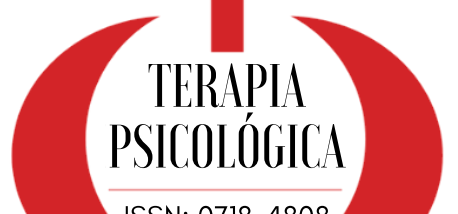

\section{Perspectivas y tendencias actuales del concepto de alienación parental: una revisión sistemática de la literatura.}

ISSN: 0718-4808

Current perspectives and trends of the concept of parental alienation: a systematic review of the literature.

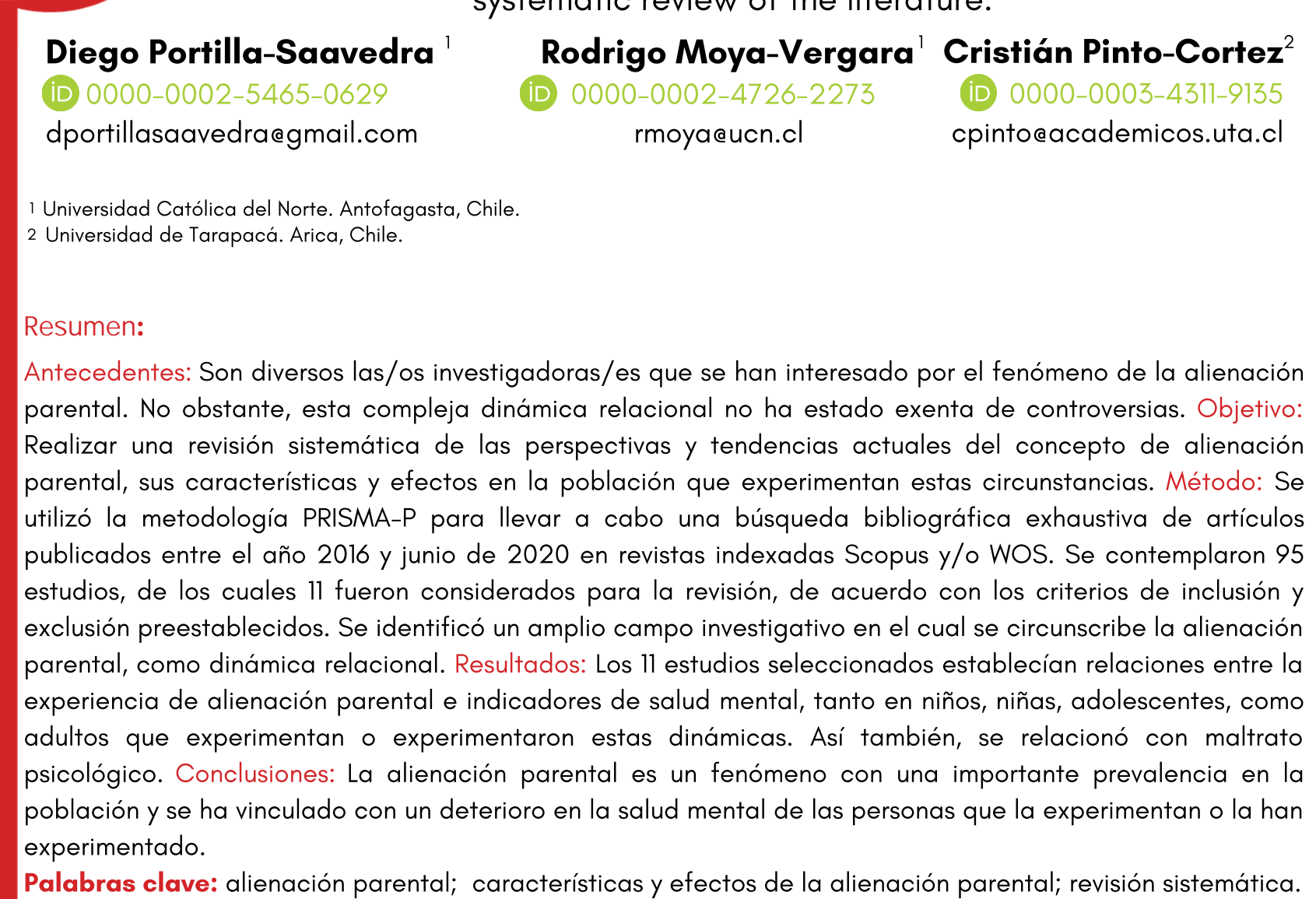

\title{
Abstract:
}

Background: Several researchers have been interested in the phenomenon of parental alienation. However, this complex relational dynamic has not been without controversy. Objective: To conduct a systematic review of current perspectives and trends on the concept of parental alienation, its characteristics and effects on the population experiencing these circumstances. Method: PRISMA-P methodology was used to conduct an exhaustive literature search of articles published between 2016 and June 2020 in Scopus and/or WOS indexed journals. Ninety-five studies were contemplated, of which 11 were considered for the review, according to the pre-established inclusion and exclusion criteria. A broad field of research was identified in which parental alienation is circumscribed as a relational dynamic. Results: The 11 selected studies established relationships between the experience of parental alienation and mental health indicators, both in children, adolescents and adults who experience or have experienced these dynamics. Thus, it was also related to psychological maltreatment. Conclusions: Parental alienation is a phenomenon with a significant prevalence in the population and has been linked to a deterioration in the mental health of people who experience or have experienced it.

Keywords: parental alienation; characteristics and effects parental alienation; systematic review. 


\section{Introducción}

A través de los años, diversos investigadores/as han tratado de situar los conflictos entre los progenitores y su repercusión en el bienestar y la salud mental de la población infantojuvenil (Demby, 2016; Kruk, 2018; Mctavish et al., 2016; Mercer, 2019; Reay, 2015). En este sentido, se ha destacado cómo los conflictos entre progenitores en la separación y el divorcio pueden transformarse en uno de los principales factores de riesgo para los niños, niñas y adolescentes que se encuentran inmersos/as en estas dinámicas, inclusive, considerándose como experiencias de carácter traumático (Duerr y Hautzinger, 2019; Harold y Sellers, 2018; Visser et al., 2017). Los niños, niñas y adolescentes expuestos/as a la violencia relacional de los progenitores están en riesgo significativo de múltiples resultados negativos, incluyendo depresión, agresión, trastornos de conducta, violencia, abuso de sustancias, fracaso académico, trastorno de estrés postraumático y tendencias suicidas (Corrás et al., 2017; McTavish et al., 2016; Van der Wal et al., 2019). Harold et al. (2012) demostraron que los niños que mantienen problemas de conductas antisociales provienen de familias con conflictos entre progenitores. Además, ante estas circunstancias, la capacidad de los progenitores de ofrecer a sus hijos/a seguridad y protección, puede verse importantemente disminuida, afectándose las relaciones materno y paterno filiales (Demby, 2016).

En este marco, Gardner (1985) fue uno de los autores pioneros en estudiar la conflictividad parental en el divorcio y en contexto de disputa por la custodia. Este autor acuñó el denominado síndrome de alienación parental (SAP), el que se centraba en develar cómo los conflictos por la custodia podían devenir en el rechazo de un niño/a al contacto con uno de sus progenitores. Para este autor, el SAP reflejaba un "lavado de cerebro" de un progenitor/a alienante con respecto al hijo/a en común con el progenitor/a objetivo, generando una campaña de denigración en relación con este último. Por progenitor objetivo, Gardner (2002) aludía a la madre o padre que es receptor/a de las dinámicas de alienación, por parte del otro/a progenitor/a. Este autor, situaba esta dinámica generalmente en contexto de disputa de la custodia de niños, niñas y adolescentes y que se caracterizaba por la existencia de una constelación de síntomas presentes en el niño o niña, tales como: Una campaña de denigración con respecto al progenitor/a objetivo, débiles o absurdas racionalizaciones para la desaprobación, ausencia de ambivalencia, el fenómeno del pensador/a independiente, apoyo reflexivo al progenitor/a alienante en el conflicto parental, ausencia de culpa por crueldad y/o explotación del progenitor/a alienado y la propagación de la alienación a la familia extendida (Gardner, 2002). No obstante, su teoría no estuvo exenta de controversias, por la no inclusión del SAP en el Manual de Diagnóstico y Estadístico de los Trastornos Mentales de la Asociación Americana de Psiquiatría (Vilalta y Winberg, 2017). Por otra parte, O'Donohue et al. (2016) postulan que no existen estudios sólidos y revisados por pares relacionados con los conceptos, ideas o fundamentos descritos por el "síndrome de alienación parental" (SAP) de Gardner. Kelly y Johnston (2001) criticaron el constructo del SAP por enfocarse exclusivamente en el progenitor o progenitora alienante como agente etiológico de 
los "síntomas" del niño, niña o adolescente. Estas autoras afirman que "la investigación muestra que, dentro del divorcio de alto conflicto, ambos progenitores pueden comportarse de manera tal que perjudiquen la relación del niño/a con el otro progenitor/a" (O'Donohue et al., 2016, p. 116). Pese a las distintas críticas sobre el SAP, Vilalta y Winberg (2017) sostienen que Gardner hizo énfasis en que, para el diagnóstico de alienación parental debía predominar un descarte previo de circunstancias que en efecto constituyan vulneraciones con respecto a niños, niñas y adolescentes por parte de dicho/a progenitor/a. De acuerdo con lo planteado por estos autores, no se configura como un fenómeno que avale o sustente el abuso sexual infantil o la violencia de género, dado que son excluyentes.

A raíz de las críticas y controversias a la teoría, el curso de la investigación sobre este fenómeno en particular ha optado por reformular los postulados de Gardner (1985) y, por consiguiente, adoptar el concepto de "alienación parental" como de interés científico. En los estudios disponibles, se encuentran innumerables definiciones de alienación parental, teniendo como factor común, el reconocimiento de una problemática profunda, de alto impacto en las familias y por sobre todo en población infantojuvenil. Por ejemplo, Jaffe et al. (2017) plantean que la alienación parental alude a un constructo que describe una campaña de privación de derechos de los niños/as por parte de uno/a de los progenitores contra el/la otro/a, específicamente durante el divorcio. Además, un estudio de prevalencia encontró que más de una cuarta parte de las/os adultos/as de una muestra, informaban haber sufrido alienación parental (Baker, 2009). En relación con esto, el impacto de la alienación parental en los niños, el/la progenitor/a objetivo y todo el sistema familiar es sustancial (Harman et al., 2019), no obstante, requiere de ciertas consideraciones. Por consiguiente, Baker(2020) planteó el modelo de cuatro factores de alienación parental, el cual describe que debiesen presentarse al menos cuatro condiciones para que exista la alienación parental: (1) una relación positiva previa entre el/la niño/a y el/la progenitor/a ahora rechazado/a; (2) ausencia de maltrato por parte del progenitor/a rechazado/a; (3) uso de conductas alienantes por parte del progenitor/a favorecido/a; y (4) presencia de manifestaciones conductuales de alienación en el niño o niña.

En el último tiempo, la alienación parental ha sido catalogada como una forma grave de abuso emocional o maltrato infantil (Kruk, 2018). Se ha postulado que es concordante con definiciones clásicas de maltrato infantil, lo que repercute en la necesidad de otorgar a este fenómeno, protagonismo en términos sociales y de protección (Harman et al., 2018).

A partir de la revisión efectuada, se denotaron distintos vacíos en la literatura, en específico se encontraron escasos artículos revisados por pares y actualizados sobre la temática de alienación parental a nivel hispanoparlante, lo que también se explica por la adopción de nuevas nomenclaturas para este fenómeno, por parte de los/as investigadores/as de esta lengua. 
El presente artículo propone revisar la literatura disponible en los últimos cinco años con respecto a la alienación parental, sus características, efectos en niños, niñas, adolescentes, adultos/as y las perspectivas actuales en investigación sobre este fenómeno relacional. El énfasis en estas dinámicas relacionales y sus efectos en la población de niños, niñas, adolescentes y adultos/as puede contribuir a visibilizar una problemática de afectación directa a la salud mental de dicha población, como también, a desarrollar elementos teóricos que permitan profundizar a futuro, en líneas de investigación símiles. La presente revisión se basa en tres preguntas: ¿Qué refiere la literatura sobre alienación parental? ¿Qué características y efectos tiene la alienación parental en la población de niños, niñas, adolescentes y adultos/as? ¿Cuáles son las principales tendencias de investigación en alienación parental? Estas preguntas se desarrollan por subtemas en el respectivo apartado de resultados.

\section{Método}

La presente revisión sistemática ha sido realizada 'teniendo en consideración las recomendaciones de la guía PRISMA (preferred reporting items for systematic reviews and Meta-analyses) (Moher et al., 2015; Shamseer et al., 2015).

\section{Revisión bibliográfica}

Los estudios fueron seleccionados a través de las bases de datos Scopus y WOS. Se inició la búsqueda entre marzo de 2020 y junio de 2020. Los términos de búsqueda utilizados fueron: 'parental alienation', 'alienación parental', 'characteristics of parental alienation', 'características alienación parental', 'effects of parental alienation' y 'efectos alienación parental'.

\section{Criterios de inclusión}

Los criterios de selección de los documentos fueron: 1) ser un estudio cuantitativo o cualitativo publicado en una revista científica; 2) tener como idioma el inglés o español; 3) encontrarse comprendido en el rango de años 2016 y junio de 2020; 4) haber incluido cualquier tipo de población (niños, niñas, adolescentes y/o adultos/as).

\section{Selección de estudios}

El proceso de selección de estudios se focalizó en analizar artículos científicos en los cuales se desarrollase la alienación parental en el período definido por los criterios de inclusión. Este lapso se consideró en función de velar por una revisión de las ultimas perspectivas y tendencias asociadas a la alienación parental, en cuanto al uso del concepto en diversos 
estudios, en la caracterización, los efectos y las principales tendencias.

Una vez recuperados todos los trabajos se procedió a su revisión con el objetivo de corroborar cuáles cumplían los criterios de inclusión, los cuales fueron evaluados de forma exhaustiva con el propósito de extraer la información pertinente. En la etapa inicial, se revisaron títulos y resúmenes de las referencias recolectadas. Por consiguiente, se revisaron los textos de forma íntegra en los casos en los cuales se cumplían los criterios preestablecidos. La información obtenida fue codificada en una base de datos para su posterior análisis y discusión.

Como criterio de exclusión, se descartaron artículos que se encontrarán fuera del intervalo de años referido, con la finalidad de abogar por una revisión actualizada de las últimas tendencias sobre los conceptos en cuestión. De igual manera, se desestimaron artículos que hacían alusión a temas similares, pero no íntegramente relacionados con las preguntas de la revisión, como los que se referían al análisis de casos en distintas cortes judiciales a lo largo el mundo, o la perspectiva legal del concepto de alienación parental de acuerdo con la legislación vigente.

\section{Resultados}

\section{Características de los estudios}

Se seleccionaron once artículos para la presente revisión (figura 1). Con relación al origen de los estudios, cinco fueron llevados a cabo en Estados Unidos (Aloia y Strutzenberg, 2019; Bernet et al., 2018; Bernet et al., 2020; Harman et al., 2016; Rowlands, 2019); tres en Italia (Baker y Verrocchio, 2016; Verrocchio et al., 2018; Verrocchio et al., 2019); tres en Australia (Balmer et al., 2018; Bentley y Matthewson, 2020; Lee-Maturana et al., 2020). Dos estudios revisados fueron dirigidos con muestra que consideraba a niños, niñas y adolescentes con un promedio de edad de trece años (Bernet et al., 2018; Bernet et al., 2020). Al menos seis estudios consideraban adultos/as que reportaban haber estado expuestos/as a alienación parental en etapas anteriores (infancia y/o adolescencia) (Aloia y Strutzenberg, 2019; Baker y Verrocchio, 2016; Bentley y Matthewson, 2020; Harman et al., 2016; Verrocchio et al., 2018; Verrocchio et al., 2019). Tres estudios se llevaron a cabo con adultos/as que se consideraban a sí mismos/as como alienados/as de sus hijos o hijas, vale decir, estudios que desarrollaban la perspectiva del progenitor/a alienado/a (Balmer et al., 2018; Lee-Maturana et al., 2019; Rowlands, 2019). 


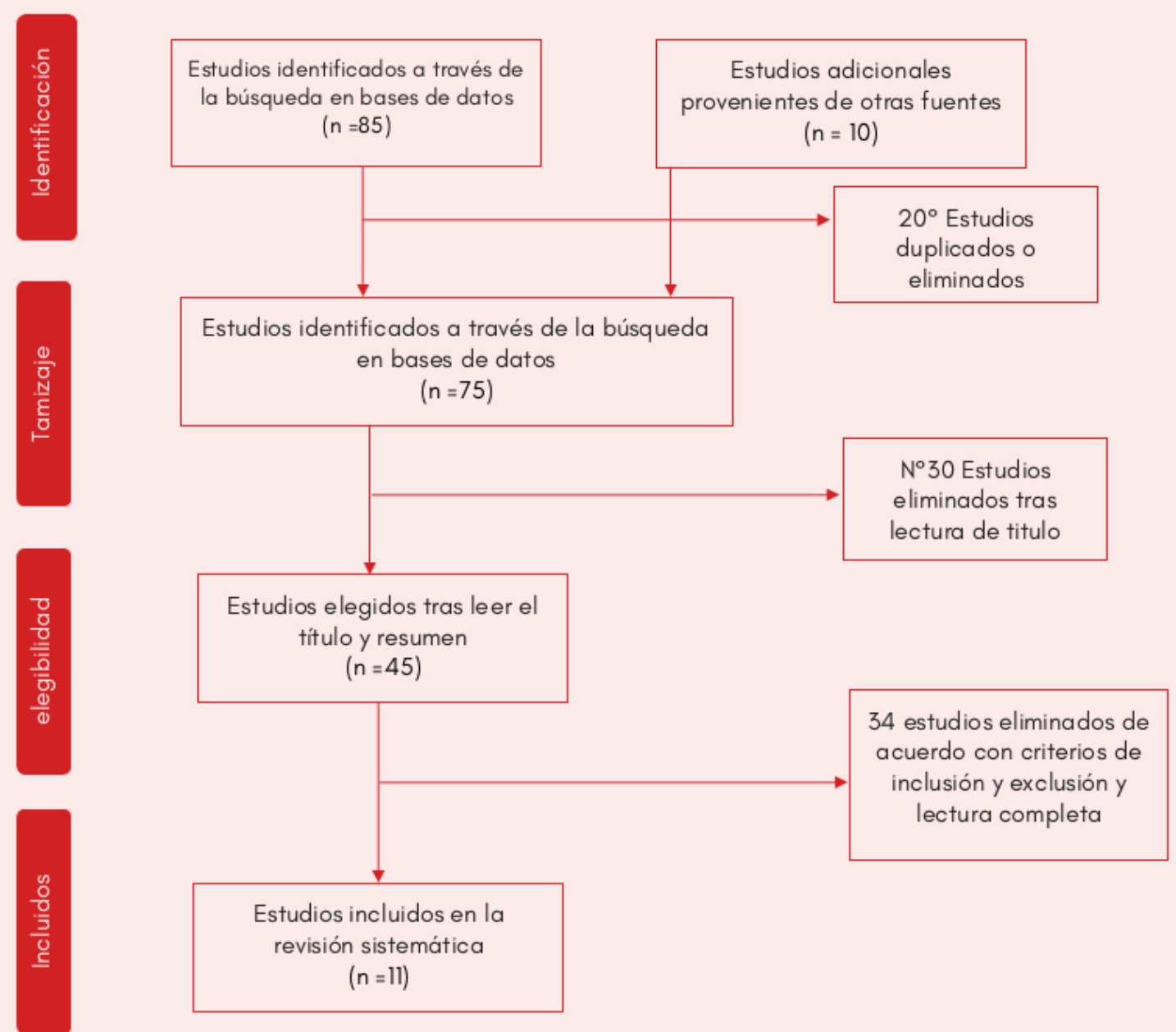

Nota: Descripción del proceso de selección de estudios para la revisión.

Figura 1: Proceso de selección de los estudios (revisión sistemática de artículos publicados entre 2016 y junio de 2020).

\section{Tendencias en investigación sobre alienación parental}

En el intervalo de años revisado se observa un distanciamiento en la investigación en alienación parental en términos de establecer definiciones al fenómeno. En efecto, en desmedro de la búsqueda de esta conceptualización, se ha mostrado interés en el desarrollo de investigaciones en áreas claramente definidas. En este sentido, al menos tres estudios de la presente revisión tenían como objetivo el construir y validar instrumentos de corte psicométrico, tanto con población de niños, niñas y adolescentes, como adultos que informaban haber estado alienados/as (Bernet et al., 2018; Bernet et al., 2020; Rowlands, 2020). Por otra parte, dos estudios se centraban en indagar la perspectiva del progenitor o progenitora objetivo de alienación parental (Balmer et al., 2018; Lee-Maturana et al., 2020). Por último, cinco estudios presentaban investigaciones de corte retrospectivo con adultos/as que han padecido estas experiencias durante la infancia $y / 0$ adolescencia (Aloia y Strutzenberg, 2019; Baker y Verrocchio, 2016; Bentley y Matthewson, 2020; Verrocchio et al., 
2018; Verrocchio et al., 2019).

En lo que concierne a la tipología de estudio, nueve estudios incluyeron métodos cuantitativos (Aloia y Strutzenberg, 2019; Baker y Verrocchio, 2016; Balmer et al., 2018; Bernet et al., 2018; Bernet et al., 2020; Harman et al., 2016; Rowlands, 2019; Verrocchio et al., 2018; Verrocchio et al., 2019) y al menos dos estudios fueron de corte cualitativo (Bentley y Matthewson, 2020; Lee-Maturana et al., 2020).

Cuatro investigaciones relacionaron la alienación parental con medidas de salud mental como depresión, ansiedad, calidad de vida, baja autoestima (Aloia y Strutzenberg, 2019; Baker y Verrocchio, 2016; Verrocchio et al., 2018; Verrocchio et al., 2019). No obstante, en la totalidad de los estudios revisados, se consignaba en los respectivos apartados introductorios las consecuencias negativas en términos de salud mental de la alienación parental en las personas que la han experimentado.

\section{Conceptualización actual}

Son insoslayables las controversias históricas que circunscriben al concepto de alienación parental, no obstante, para la presente revisión se enfatizó en aquellas definiciones más recientes utilizadas en diversos estudios comprendidas en el rango de tiempo preestablecido, a fin de delimitar cuales son las últimas tendencias y perspectivas en su utilización. En este sentido, los once estudios seleccionados para la presente revisión conceptualizan el fenómeno de alienación parental. Por ejemplo, el estudio de Baker y Verrocchio (2016) caracteriza la alienación parental, como una dinámica familiar en la que uno/a de los progenitores (el progenitor/a alienante) se involucra en el uso de comportamientos específicos que podrían resultar en el rechazo injustificado de un niño o niña con respecto del otro progenitor/a (el progenitor/a objetivo). Otros investigadores/as han complementado esta conceptualización en donde se le ha definido como un proceso de influencia negativa sobre un niño o niña por parte de uno de los progenitores destinado a excluir y aislar al otro progenitor/a (Aloia y Strutzenberg, 2019; Balmer et al., 2018).

Por otra parte, Bernet et al. (2018) conceptualiza la alienación parental como una condición mental en la que los niños/as, generalmente niños/as cuyos progenitores están involucrados en una separación de alto conflicto o divorcio, se alían fuertemente con uno de los progenitores (el preferido o progenitor/a alienante) y rechazan la relación con el otro progenitor/a (el/a progenitor/a rechazado/a u objetivo) sin justificación legítima. Posteriormente, otra investigación similar define la alienación parental como el rechazo de un progenitor/a sin justificación legítima por parte de un niño o niña, diferenciándolo del concepto de "distanciamiento realista", que implica el rechazo de un/a progenitor/a por una 
buena razón. En este estudio se hace énfasis en que ambos conceptos generalmente son aceptados entre los profesionales de la salud mental y jurídicos (Bernet et al., 2020).

Siguiendo este marco referencial, Harman et al. (2016) diferencian la alienación parental de los comportamientos alienadores parentales. Estos últimos son definidos como acciones que un progenitor/a lleva a cabo para intencionadamente, o sin querer, distanciar a un hijo/a (o hijos/a) del del otro progenitor/a, independientemente del impacto que estos comportamientos tengan en el niño o niña.

De acuerdo con lo revisado, la alienación parental plantea múltiples concomitantes que convergen tanto en su génesis como en su mantención, siendo relevante situarla como una dinámica familiar de alta hostilidad entre progenitores posterior a la separación o al divorcio, que implica el establecimiento de coaliciones familiares que tienen como resultado el rechazo injustificado al contacto de un niño o niña con respecto a su progenitor/a.

\section{Estudios de Prevalencia}

Al menos un estudio de los revisados enfatiza en la prevalencia de este fenómeno en la población en Estados Unidos, en donde con una muestra probabilística de 610 personas se estimó que el $13,4 \%$ de los adultos/as reportó haber vivenciado algún grado de alienación parental en su etapa infantil (Harman et al., 2016). Este estudio señala ser pionero en cuantificar la prevalencia de alienación parental en la población y otorga énfasis en la necesidad de replicar investigaciones similares en otras latitudes del mundo. Pese al interés que suscita este fenómeno familiar, no se encuentran mayores estudios recientes que abarquen cifras o porcentajes de la población que haya estado expuesta a estas dinámicas, lo que representa un vacío en términos investigativos.

\section{Estudios psicométricos}

Las investigaciones de carácter psicométrico han apuntado mayormente a analizar y someter a prueba instrumentos para medir la existencia de alienación parental en contextos clínicos y jurídicos, generalmente con muestras por conveniencia. Esta corriente surge ante la carencia herramientas sólidas y confiables que permitan en efecto, la detección de esta dinámica relacional (Bernet et al., 2018; Bernet et al., 2020; Rowlands, 2020). Por ejemplo, el cuestionario sobre la aceptación y rechazo de los niños/as con respecto a sus progenitores (Child-Parental Acceptance-Rejection Questionnaire PARQ) proporciona una medida objetiva y cuantitativa de las percepciones de los niños/as en torno a la relación con sus figuras parentales (Bernet et al., 2018; Bernet et al., 2020). Esta investigación con una muestra de 151 niños, niñas y adolescentes sustenta que la alienación parental es una realidad, debido a que las/os participantes severamente alienados manifestaron consistentemente un mecanismo 
mental desadaptativo. En el caso de Rowlands (2019), esta autora realizó un análisis factorial confirmatorio del instrumento Rowlands Parental Alienation Scale (RPAS) el que fue administrado a 521 padres y madres que referían haber estado alienados/as. Este estudio analizó el modelo de seis factores por sobre el de ocho (Gardner, 1985) dispuesto en la literatura clásica de alienación parental. El modelo de seis factores incorpora: ausencia de afecto positivo, campaña de denigración, apoyo reflexivo incondicional, escenarios prestados, animosidad hacia la familia extensa y el fenómeno del pensador independiente. En este sentido, se halló que el modelo más parsimonioso era el de seis factores, debido a que se ajustaba mejor a los datos que el modelo de ocho factores. Además, todos los constructos tenían validez convergente y discriminante.

\section{Características de la alienación parental}

De acuerdo con los hallazgos encontrados, el fenómeno de alienación parental mantiene múltiples variables que inciden tanto en su génesis, como en su mantención. Por ejemplo, Balmer et al. (2017) señalan que la alienación parental es un problema legítimo y grave que van en directa afectación a niños, niñas, adolescentes, sus progenitores y al sistema familiar. De los estudios seleccionados, tres de estos describen que el proceso de divorcio o separación de alto conflicto representa una circunstancia transversal este tipo de fenómenos familiares (Bernet et al., 2018; Bernet et al., 2020; Lee-Maturana et al., 2020). Además, la alienación parental puede producirse antes, durante 0 después de la separación, especialmente cuando la custodia de los/as hijos/as está en disputa (Lee-Maturana et al., 2020). En esta línea Verrochio et al. (2018) concluyen que una vez que un progenitor/a comienza las estrategias de alienación parental, es poco probable que deje de hacerlo. Los progenitores/as parecen adecuar sus estrategias de alienación parental a la madurez y desarrollo evolutivo de sus hijos/as.

Por otra parte, en la mayoría de los estudios revisados se relaciona la alienación parental con consecuencias a nivel psicológico para la población que experimenta o ha experimentado estas dinámicas, siendo un aspecto característico de este tipo de fenómenos familiares (Aloia y Strutzenberg, 2019; Baker y Verrocchio, 2016; Balmer et al., 2017; Bentley y Matthewson, 2020; Harman et al., 2016; Lee-Maturana et al., 2020; Verrocchio et al., 2018; Verrocchio et al., 2019).

\section{Efectos de la alienación parental}

Entre los principales efectos asociados a la alienación parental, ocho de los artículos seleccionados para esta revisión, son investigaciones especificas asociadas a medir el impacto psicológico de la alienación parental en la población, desde el/la progenitor/a objetivo, los niños, niñas, adolescentes y adultos/as que experimentaron estas dinámicas. La mayoría son estudios retrospectivos en donde se ha investigado la experiencia de adultos/as 
que han reconocido haber estado expuestos/as a algún grado de alienación parental en su infancia y/o adolescencia (Aloia y Strutzenberg, 2019; Baker y Verrocchio, 2016; Bentley y Matthewson, 2020; Bernet et al., 2018; Bernet et al., 2020; Harman et al., 2016; Verrocchio et al., 2018; Verrocchio et al., 2019). Estos autores señalan que la exposición a la alienación parental en etapa infantil se asoció significativamente con efectos a corto y largo plazo concordantes con maltrato psicológico.

\section{Efectos de la alienación a corto plazo}

Si bien la totalidad de los artículos incorporados en la presente revisión reporta en el apartado introductorio el deterioro en aspectos de salud mental que podrían sufrir niños, niñas y adolescentes víctimas de alienación parental, en la presente revisión solo dos artículos aluden directamente a ello (Bernet et al., 2018; Bernet et al., 2020). Por ejemplo, en investigaciones realizadas a fin de validar el instrumento "Child-Parental AcceptanceRejection Questionnaire PARQ" se halló que la alienación parental en niños, niñas y adolescentes se relaciona con un claro mecanismo mental desadaptativo y un nivel intenso de escisión (Bernet et al., 2018). Como una continuidad de este estudio, Bernet et al. (2020) demostraron que los niños, niñas y adolescentes alienados/as tienden a exagerar, tanto la aceptación al progenitor/a alienador/a, como el rechazo al progenitor/a objetivo o alienado/a.

En las investigaciones con progenitores/as que se reconocían a sí mismos/as como alienados/as destaca lo referido por Lee-Maturana et al. (2020), quienes encontraron que la alienación parental tiene graves consecuencias para los progenitores/as objetivos incidiendo en distintas dimensiones de sus vidas. Este estudio describe al menos seis consecuencias que padecen los/as progenitores/as objetivos, las cuales pueden ser: emocionales, conductuales, financieras/laborales, cognitivas, físicas y sociales. En esta misma dirección, el estudio de Balmer et al. (2018) concluyó la existencia de una correlación positiva y significativa entre la gravedad de la exposición a las tácticas de alienación parental y la valoración del estrés y de los daños o pérdidas potenciales que puede venir en el futuro, debido a la experiencia de alienación parental en progenitores/as objetivos.

\section{Efectos de la alienación a largo plazo}

Bentley y Matthewson (2020) hallaron que la ansiedad, depresión, baja autoestima, culpa, problemas de apego, dificultad en otras relaciones y reducción o retraso en el logro educativo y profesional se atribuyeron a la experiencia de alienación parental en la infancia por parte de adultos/as que la padecieron. En los estudios revisados, la alienación parental se relacionó con una mayor probabilidad de síntomas depresivos y disminución de la calidad de vida en la etapa adulta (Verrocchio et al., 2019). Además, el estudio de Verrocchio et al. (2018) con una muestra de 361 participantes obtuvo como resultado que la exposición a alienación parental en cada período del desarrollo se asoció significativamente con maltrato psicológico. Por 
ejemplo, en la niñez y en la adolescencia, la alienación parental estaba fuertemente asociada con la experiencia de ser maltratado/a por el progenitor o progenitora que se involucra en la alienación. En este sentido, se encontró que los efectos negativos de la exposición a alienación parental en la infancia/adolescencia trae diversos resultados en la etapa adulta, tales como: depresión, ansiedad, abuso de sustancias, trastornos de conducta, disminuciones en el rendimiento académico y deterioro en la autoestima (Verrocchio et al., 2019). Aloia y Strutzenberg (2019) concluyeron que la alienación parental en la infancia se asoció positivamente con la aprensión de comunicación entre progenitores e hijos/as en la edad adulta, y esta asociación fue más débil para las personas con mayor autoestima en comparación con las personas con baja autoestima. Previamente el estudio llevado a cabo de forma retrospectiva por Baker y Verrocchio (2016) propuso analizar la asociación entre la alienación parental en la infancia con el bienestar y el desarrollo socioemocional de los niños, niñas y adolescentes. Los resultados revelaron que la exposición a la alienación parental estaba relacionada a mayores índices de ansiedad y depresión.

Finalmente, Harman et al. (2016) encontraron que las personas que informaron la presencia de alienación parental eran más propensas a reportar ser despreciadas, aterrorizadas, aisladas, explotadas o corrompidas, y negadas a responder emocionalmente, aspectos que la literatura ha definido como propios del maltrato psicológico. Además, este estudio expone que todos/as los/as participantes que informaron alienación parental, también informaron de haber sido maltratados psicológicamente.

Tabla 1: Estudios considerados para la revisión.

\begin{tabular}{|c|c|c|c|c|c|c|c|}
\hline Autor $\mathrm{y}$ año & $\begin{array}{l}\text { Diseño de } \\
\text { estudio }\end{array}$ & Objetivo de investigación & Participantes & Revista & $\begin{array}{l}\text { Instrumentos } \\
\text { utilizados }\end{array}$ & $\begin{array}{l}\text { Estrategia de } \\
\text { análisis de datos }\end{array}$ & Principales hallazgos \\
\hline \multicolumn{8}{|c|}{ Estudios con diseño cualitativo } \\
\hline $\begin{array}{l}\text { Bentley y } \\
\text { Matthewson } \\
(2020)\end{array}$ & Cualitativo & $\begin{array}{l}\text { Explorar la experiencia de } \\
\text { adultos que habian experi- } \\
\text { mentado alienación pare- } \\
\text { tal durante la infancia. }\end{array}$ & $\begin{array}{l}\text { Adultos/as, } \\
n=10\end{array}$ & $\begin{array}{l}\text { American Journal } \\
\text { of Family } \\
\text { therapy }\end{array}$ & $\begin{array}{l}\text {-Entrevista } \\
\text { semiestructurada. }\end{array}$ & $\begin{array}{l}\text { Análisis temático } \\
\text { e inductivo }\end{array}$ & $\begin{array}{l}\text { Los participantes describieron experimentar } \\
\text { abuso perpetrado por el progenitor/a } \\
\text { alienador/a. Ellos/as señalaron experimentar } \\
\text { ansiedad, depresión, baja autoestima, culpa, } \\
\text { problemas de apego, dificultad en otras } \\
\text { relaciones y reducción o retrasó el logro } \\
\text { educativo y profesional que atribuyeron a su } \\
\text { experiencia de alienación parental. Estos } \\
\text { resultados evidencian que la exposición de los } \\
\text { niños/as a la alienación parental puede tener } \\
\text { ramificaciones de por vida en su bienestar } \\
\text { psicológico. }\end{array}$ \\
\hline $\begin{array}{l}\text { Lee-Matura- } \\
\text { na et al. } \\
(2020)\end{array}$ & Cualitativo & $\begin{array}{l}\text { Describir las consecuencias } \\
\text { de la alienación de un hijo } \\
\text { e identificar las estrategias } \\
\text { de afrontamiento de los } \\
\text { padres afectados para } \\
\text { hacer frente a la aliena- } \\
\text { ción. }\end{array}$ & $\begin{array}{l}\text { Adultos/as, } \\
n=54\end{array}$ & $\begin{array}{l}\text { Journal of Child } \\
\text { and Family Stu- } \\
\text { dies }\end{array}$ & $\begin{array}{l}\text {-Entrevista semies- } \\
\text { tructurada. }\end{array}$ & $\begin{array}{l}\text { Análisis temático } \\
\text { e inductivo }\end{array}$ & $\begin{array}{l}\text { Surgieron seis subtemas que describen diferentes } \\
\text { consecuencias experimentadas por los progent- } \\
\text { tores/as objetivos debido a la alienación } \\
\text { parental: emocionales, conductuales, financieras- } \\
\text { laborales, cognitivas, fisicas y sociales. Asimismo, } \\
\text { se identificaron ocho tipos diferentes de estrate- } \\
\text { gias de afrontamiento. }\end{array}$ \\
\hline \multicolumn{8}{|c|}{ Estudios con diseños cuantitativos } \\
\hline $\begin{array}{l}\text { Aloia y Stru- } \\
\text { tzenberg } \\
\text { (2019) }\end{array}$ & Cuantitativo & $\begin{array}{l}\text { Examinar la aprensión } \\
\text { comunicativa dentro de las } \\
\text { relaciones entre progent- } \\
\text { tores e hijos/as en función } \\
\text { de la alienación parental y } \\
\text { la autoestima. }\end{array}$ & $\begin{array}{l}\text { Adultos/as, } \\
n=211\end{array}$ & $\begin{array}{l}\text { Communication } \\
\text { Reports }\end{array}$ & $\begin{array}{l}\text {-Baker Strategy } \\
\text { Questionnaire } \\
\text {-Child-Parent Com- } \\
\text { munication Appre- } \\
\text { hension Measure } \\
\text {-Rosenberg's Self- } \\
\text { Steem Scale }\end{array}$ & $\begin{array}{l}\text {-Prueba } t \\
\text {-Regresión múlti- } \\
\text { ple jerárquica }\end{array}$ & $\begin{array}{l}\text { La alienación parental en la infancia se asoció } \\
\text { positivamente con la aprensión de comunicación } \\
\text { entre progenitores e hijos en la edad adulta, y } \\
\text { esta asociación fue más débil para las personas } \\
\text { con mayor autoestima en comparación con las } \\
\text { personas con baja autoestima. }\end{array}$ \\
\hline $\begin{array}{l}\text { Baker y Ver- } \\
\text { rocchio } \\
\text { (2016) }\end{array}$ & Cuantitativo & $\begin{array}{l}\text { Examinar las asociociones } \\
\text { entre la exposición a las } \\
\text { conductas de alienación } \\
\text { parental, la ansiedad y } \\
\text { depresión en una muestra } \\
\text { comunitaria de adultos } \\
\text { italianos. }\end{array}$ & $\begin{array}{l}\text { Adultos/as, } \\
n=533\end{array}$ & $\begin{array}{l}\text { American Journal } \\
\text { of Family } \\
\text { Therapy }\end{array}$ & $\begin{array}{l}\text {-Baker Strategy } \\
\text { Questionnaire (BSO) } \\
\text {-State-Trait Anxiety } \\
\text { Inventory - Form Y } \\
\text { (STALYY) } \\
\text {-Beck Depression } \\
\text { Irventory - II (BDH-II) }\end{array}$ & $\begin{array}{l}\text {-Prueba } t \\
\text {-Regresión lineal } \\
\text { por pasos }\end{array}$ & $\begin{array}{l}\text { Los resultados revelaron que la exposición a la } \\
\text { alienación parental estaba asociada a mayores } \\
\text { indices de ansiedad y depresión. Los datos se } \\
\text { suman al creciente cuerpo de conocimientos } \\
\text { sobre el impacto negativo a largo plazo de la } \\
\text { exposición a la alienación parental. }\end{array}$ \\
\hline
\end{tabular}


Perspectivas y tendencias actuales del concepto de alienación parental: una revisión sistemática de la literatura.

\begin{tabular}{|c|c|c|c|c|c|c|c|}
\hline $\begin{array}{l}\text { Bernet et al. } \\
\text { (2018) }\end{array}$ & Cuantitativo & $\begin{array}{l}\text { Evaluar la utilidad del } \\
\text { Cuestionario de acept- } \\
\text { ación y rechazo de los } \\
\text { padres (PARO) para } \\
\text { identificar y cuantificar el } \\
\text { grado de escisión, que } \\
\text { puede ayudar a diag- } \\
\text { nosticar la alienación } \\
\text { parental. }\end{array}$ & $\begin{array}{l}\text { Niños riñas y } \\
\text { adolescentes, } \\
\text { n=llo }\end{array}$ & $\begin{array}{l}\text { Journal of } \\
\text { forensic sciences }\end{array}$ & $\begin{array}{l}\text {-The Parental Accep- } \\
\text { tance-Rejection } \\
\text { Questionnaire(PARO) }\end{array}$ & -Prueba $t$ & $\begin{array}{l}\text { Los resultados mostraron que los niños severa- } \\
\text { mente alienados se involucraron en un alto nivel } \\
\text { de escisión, percibiendo al progenitor/a prefe- } \\
\text { rido/a en términos extremadamente positivos y al } \\
\text { progenitor/a rechazado/a en términos extrema- } \\
\text { damente negativos. El PARO puede ser útil tanto } \\
\text { para los clinicos como para los forenses en la } \\
\text { evaluación de los hijos de progenitores divorcia- } \\
\text { dos cuando existe una preocupación por el posi- } \\
\text { ble diagnóstico de alienación parental. }\end{array}$ \\
\hline $\begin{array}{l}\text { Bernet ef al. } \\
(2020)\end{array}$ & Cuantitativo & $\begin{array}{l}\text { La hipótesis de este estudio } \\
\text { fue que una prueba } \\
\text { psicológica, el cuestionario } \\
\text { de aceptación y rechazo } \\
\text { de los progenitores (PARO), } \\
\text { ayudará a distinguir a los } \\
\text { niños/as severamente } \\
\text { alienados de los no } \\
\text { alineados. }\end{array}$ & $\begin{array}{l}\text { Niños riñas y } \\
\text { adolescentes, } \\
n=116\end{array}$ & $\begin{array}{l}\text { Journal of foren- } \\
\text { sic sciences }\end{array}$ & $\begin{array}{l}\text {-The Child Version of } \\
\text { the Parental Accep- } \\
\text { tance-Rejection } \\
\text { Questionnaire: Mo- } \\
\text { ther and Father } \\
\text { Forms }\end{array}$ & -Anova & $\begin{array}{l}\text { Usando un Puntaje PARQ-Gap de } 90 \text { como punto } \\
\text { de corte, esta prueba fue } 99 \% \text { pre cisa para dist in- } \\
\text { guir a los/as niños/as severamente alienados de } \\
\text { los no a lienados. }\end{array}$ \\
\hline $\begin{array}{l}\text { Balmer et al. } \\
\text { (2018) }\end{array}$ & Cuantitativo & $\begin{array}{l}\text { Determinar las experiencias } \\
\text { especificas de los progeni- } \\
\text { tores sobre la alienación } \\
\text { parental posterior a la se- } \\
\text { paración del progenitor/a } \\
\text { alienante, e investigar las } \\
\text { caracteristicas comunes de } \\
\text { los progenitores objetivo. }\end{array}$ & $\begin{array}{l}\text { Adultos/as, } \\
n=179\end{array}$ & $\begin{array}{l}\text { Australian journal } \\
\text { of psychology }\end{array}$ & $\begin{array}{l}\text {-The stress appraisal } \\
\text { meosure } \\
\text {-The Depression, An- } \\
\text { xiety, and Stress } \\
\text { Scale (DASS-21) } \\
\text {-The Parenting Sense } \\
\text { of Competence Sca- } \\
\text { le } \\
\text {-The Parent-Child } \\
\text { Relationship Inven- } \\
\text { tory } \\
\text {-Cuestionario sobre } \\
\text { exposición a aliena- } \\
\text { ción parental elabo- } \\
\text { rado por las/os in- } \\
\text { vestigadoras/es. }\end{array}$ & $\begin{array}{l}\text {-Regresión lineal } \\
\text {-Anova }\end{array}$ & $\begin{array}{l}\text { Los resultados indicaron la gravedad del impacto } \\
\text { de la exposición a la alienación parental para los } \\
\text { progenitores seleccionados, y destacaron la } \\
\text { necesidad de una investigación empírica sobre la } \\
\text { eficacia de las intervenciones y los servicios de } \\
\text { apoyo para ayudar a los/as progenitores } \\
\text { objetivos. }\end{array}$ \\
\hline $\begin{array}{l}\text { Harman et } \\
\text { d. (2016) }\end{array}$ & Cuantitativo & $\begin{array}{l}\text { Determinar la prevalencia } \\
\text { de la alienación parental. }\end{array}$ & $\begin{array}{l}\text { Adultos/as, } \\
\qquad n=610\end{array}$ & $\begin{array}{l}\text { Children and } \\
\text { Youth Services } \\
\text { Review }\end{array}$ & $\begin{array}{l}\text {-Cuestionario sobre } \\
\text { exposición a aliena- } \\
\text { ción parental elabo- } \\
\text { rado por las/os in- } \\
\text { vestigadoras/es. }\end{array}$ & أل cuadrado & $\begin{array}{l}\text { Los hallazgos sugieren que decenas de millones } \\
\text { de adultos/as y sus hijos/as pueden verse afec- } \\
\text { tados por la alienación parental, que es mucho } \\
\text { mayor que las estimaciones anteriores. Además, } \\
\text { los resultados muestran evidencia de alienación } \\
\text { parental en todos los estratos socioeconómicos. }\end{array}$ \\
\hline $\begin{array}{l}\text { Verroochio } \\
\text { et al. (2018) }\end{array}$ & Cuantitativo & $\begin{array}{l}\text { Determinar la relación } \\
\text { entre exposición a la } \\
\text { alienación parental (AP) en } \\
\text { diferentes momentos de } \\
\text { desarrollo periodos y el } \\
\text { maltrato psicológico. }\end{array}$ & $\begin{array}{l}\text { Adultos/as, } \\
\quad n=395\end{array}$ & $\begin{array}{l}\text { Journal of Family } \\
\text { Therapy }\end{array}$ & $\begin{array}{l}\text {-Cinco items de la } \\
\text { escala "Emotional } \\
\text { Neglect Scale"-Cin- } \\
\text { co items de la escala } \\
\text { emocional de la es- } \\
\text { cala "Childhood } \\
\text { Trauma". } \\
\text {-Siete items de la } \\
\text { subescala de mal- } \\
\text { trato de la Child } \\
\text { Abuse and Trauma } \\
\text { Scale. } \\
\text {-Cinco items de la } \\
\text { subescala de Con- } \\
\text { flict Tactics Survey. }\end{array}$ & -Regresión lineal & $\begin{array}{l}\text { Los resultados revelaron que la exposición a la } \\
\text { alienación parental en cada periodo de tiempo se } \\
\text { asoció significativamente con maltrato psi- } \\
\text { cológico. Los hallazgos sugirieron que una vez } \\
\text { que se inició la alienación parental por un/a } \\
\text { progenitor/a era muy probable que continuara. } \\
\text { Se describe la intratabilidad de alienación } \\
\text { parental se ha descrito en la literatura clínica. }\end{array}$ \\
\hline $\begin{array}{l}\text { Verrocchio } \\
\text { et al. }(2019)\end{array}$ & Cuantitativo & $\begin{array}{l}\text { Examinar la relación entre } \\
\text { la exposición a la aliena- } \\
\text { ción parental, la depresión } \\
\text { y calidad de vida rela- } \\
\text { cionada con la salud en } \\
\text { adultos italianos. }\end{array}$ & $\begin{array}{l}\text { Adultos/as, } \\
n=515\end{array}$ & $\begin{array}{l}\text { Health and Qua- } \\
\text { lity of Life Out- } \\
\text { comes }\end{array}$ & $\begin{array}{l}\text {-Baker Strategy } \\
\text { Questionnaire (BSO) } \\
\text {-Beck Depression } \\
\text { Inventory- II (BDI-II) } \\
\text {-Short-Form } 36 \text { (SF- } \\
\text { 36) health survey }\end{array}$ & -ANCOVA & $\begin{array}{l}\text { La exposición infantil a la AP se relacionó con una } \\
\text { mayor probabilidad de sintomas depresivos y } \\
\text { disminuyó la calidad de vida en la edad adulta. } \\
\text { Los hallozgos sugieren la necesidad de } \\
\text { intervenciones preventivas y clinicas para } \\
\text { proteger a los niños/as vulnerables involucrados } \\
\text { en la alienación parental. }\end{array}$ \\
\hline $\begin{array}{l}\text { Rowlands } \\
\text { (2019) }\end{array}$ & Cuantitativo & $\begin{array}{l}\text { Evaluar la estructura } \\
\text { factorial de la Escala de } \\
\text { Alienación Parental de } \\
\text { Rowlands (RPAS). }\end{array}$ & $\begin{array}{l}\text { Adultos/as, } \\
n=521\end{array}$ & $\begin{array}{l}\text { Journal of divor- } \\
\text { ce and remar- } \\
\text { riage }\end{array}$ & $\begin{array}{l}\text {-The Rowlands Po- } \\
\text { rental Alienation } \\
\text { Scale (RPAS) }\end{array}$ & $\begin{array}{l}\text {-Aná lisis factorial } \\
\text { confirmat orio }\end{array}$ & $\begin{array}{l}\text { Se concluyó que el modelo de seis factores de } \\
\text { alienación parental, era más parsimonioso y se } \\
\text { ajustaba mejor a los datos que el modelo de ocho } \\
\text { factores, clásico de la literatura de alienación } \\
\text { parental. }\end{array}$ \\
\hline
\end{tabular}

Nota. Se enumeran los estudios (11) considerados para la presente revisión especificando sus autores, la metodología, objetivo, muestra, revista de publicación, instrumentos utilizados, estrategia de análisis de datos y principales hallazgos.

\section{Discusión}

El objetivo de la presente investigación fue realizar una revisión sistemática de la literatura para identificar y sintetizar la información relevante disponible en las investigaciones sobre alienación parental entre el año 2016 y junio de 2020.

Como constatan los diversos trabajos publicados al respecto, la alienación parental es un fenómeno familiar de alto interés en el escenario científico. Es así, que para la presente revisión se hallaron más de noventa y cinco trabajos en revistas indexadas Scopus y WOS, lo 
que refleja la motivación de las/os investigadoras/es por profundizar en la temática.

A raíz de la revisión llevada a cabo, se pudo corroborar que predominantemente los estudios e investigaciones más recientes sobre el fenómeno han privilegiado definiciones y usos más clásicos del concepto de alienación parental, tal como lo define Lorandos et al. (2013) quien refiere que estas circunstancias implican una condición psicológica en la que un niño/a se alía fuertemente con un progenitor/a alienante (o preferido) y rechaza una relación con el/la progenitor/a alienado/a (u objetivo) sin justificación legítima. No obstante, se evidencia un interés creciente en amplificar y diversificar las muestras con las cuales se ha desarrollado investigación acerca de este fenómeno. En este sentido, la alienación parental ha sido estudiada tanto en muestras compuestas por niños, niñas, adolescentes y adultos/as que experimentan o experimentaron estas dinámicas en su infancia, como también por adultos/as objetivos de la misma (Aloia y Strutzenberg, 2019; Baker y Verrocchio, 2016; Balmer et al., 2018; Bernet et al., 2018; Bernet et al., 2020; Harman et al., 2016; Rowlands, 2019; Verrocchio et al., 2018; Verrocchio et al., 2019). Por otra parte,se han conducido investigaciones sobre este fenómeno en diversas latitudes del mundo, por ejemplo, Australia, Estados Unidos e Italia (Aloia y Strutzenberg, 2019; Baker y Verrocchio, 2016; Balmer et al., 2018; Bernet et al., 2018). Por otra parte, los hallazgos de la presente revisión son consistentes en relacionar la experiencia de alienación parental con repercusiones en la salud mental, que se traducen en un deterioro en la autoestima, depresión, ansiedad, problemas de rendimiento académico y/o laboral (Aloia y Strutzenberg, 2019; Baker y Verrocchio, 2016; Verrocchio et al., 2018; Verrocchio et al., 2019). Además, no solo se verían afectados niños, niñas y/o adolescentes (Bernet et al., 2018; Bernet et al., 2020), sino también, adultos que experimentaron estas dinámicas relacionales en su infancia, lo que ha estudiado desde los efectos a largo plazo (Baker y Verrocchio, 2016; Verrocchio et al., 2018; Verrocchio et al., 2019). De igual forma se ha investigado con progenitores/as que son objeto de estas dinámicas, lo que ha reflejado que estas personas de igual manera evidencian un deterioro en áreas claves del funcionamiento psicosocial (emocional, conductual, financiero/laboral, cognitiva, física y social) (Balmer et al., 2018; Lee-Maturana., 2020). Estos hallazgos son ampliamente concordantes con la literatura de opinión sobre alienación parental, que ha calificado esta experiencia como una forma de maltrato infantil o abuso emocional (Harman et al., 2018; Harman et al., 2019; Kruk, 2018).

En este punto, cabe destacar que la alienación parental abarca una dinámica relacional compleja, que no incorpora exclusivamente a progenitores y sus hijos/as, sino también a miembros de la familia extensa, profesionales del área sanitaria, jueces de familia, abogados/as (Baker, 2020; Johnston y Sullivan, 2020; Warshak, 2020). No obstante, son escasos los estudios de la presente revisión que realicen la integración de estos aspectos, pese a enunciarse mayormente en el respectivo apartado del marco teórico. Este vacío de la literatura es concordante con lo propuesto por autores como Johnston y Sullivan (2020), quienes postulan que mayoritariamente la evidencia propone modelos comprensivos de factor 
único para este fenómeno, por sobre modelos de corte multifactorial. Vale decir, que en la alienación parental existe una relación causal primaria entre el comportamiento alienante del progenitor/a y la respuesta de rechazo del niño/a con respecto al progenitor/a objetivo. En los hallazgos se encontró que gran parte de los artículos seleccionados consideraban esta dimensión única del fenómeno, aspectos que podrían coartar y limitar la profundidad de los resultados. En este sentido, diversos autores/as han propuesto en artículos teóricos los modelos de corte multifactorial. Estos modelos describen que la alienación parental no tendría como única causal etiológica, las acciones de un/a progenitor/a alienante, sino más bien, estarían relacionadas con aspectos psicológicos y la parentalidad de ambos progenitores, su historial de pareja, la intensidad del divorcio, la injerencia de familias extensas y profesionales sanitarios, jurídicos y sociales (Johnston y Sullivan, 2020; Warshak, 2020).

Por otra parte, pese a que la presente revisión tenía como criterio de inclusión el incorporar artículos que estuviesen escritos en inglés y en español, todos los artículos revisados y seleccionados, fueron escritos en inglés. Esto podría tener explicación en que las/os autores en la literatura española han buscado distanciarse mayoritariamente de la teoría primigenia de alienación parental, es decir, la de Gardner (Vilalta y Winberg, 2017). En este sentido, han optado por otras nomenclaturas asociadas al concepto de alienación parental, como son las interferencias parentales (Antón y 2019; González, 2017) y las prácticas alienadoras familiares (Linares, 2015). Por ejemplo, González (2017) define las interferencias parentales como dinámicas que implican comportamientos que dañan la relación de un niño/niña con uno de sus progenitores y lo diferencia de la alienación parental, dado que esta última, sería el resultado de la sistematicidad de la interferencia. Por otro lado, Linares (2015) propone una alternativa al Síndrome de Alienación Parental, al referir las practicas alienadoras familiares como un conjunto de situaciones relacionales caracterizadas por la existencia de un manipulador/a exitoso (el alienador) y manipulador/a fracasado (el alienado), la participación de hijos e hijas y familias extensas, y por último, la estimulación del conflicto por parte de profesionales terceros (jueces, psicólogos/as, abogados/as). Ambas perspectivas parecen coincidir en la necesidad de otorgar una mirada mayormente ecológica a este fenómeno, aspectos que también ya se han explicitado por otros/as investigadores de habla inglesa (Johnston y Sullivan, 2020; Warshak, 2020).

Si bien parece existir consenso en la existencia del fenómeno de alienación parental, las controversias sobre la temática han estado arraigadas históricamente a la unificación en la definición del concepto, lo que no ha permitido avanzar científicamente, como en otras áreas temáticas afines.

Este estudio presenta algunas limitaciones, cabe destacar que al centrar la atención con respecto a artículos de revistas WOS y Scopus, se desestimó la literatura conformada por revisión de temas, congresos, artículos, simposios, ensayos, que de igual manera mantienen validez y que pueden añadir y sumar nuevas perspectivas a la discusión actualizada sobre alienación parental. Por otra parte, de la evidencia disponible en torno a la temática, existen 
abundantes artículos que profundizan en la complejidad conceptual y teórica de la alienación parental, poniendo en perspectiva las múltiples definiciones que circunscriben a este fenómeno familiar en particular. Esto conlleva el hecho de hallar un sinnúmero de definiciones, dificultándose la homogeneización de la información sobre el fenómeno, en cuanto a una definición precisa y uniforme, como acontece con otras temáticas afines.

\section{Conclusión}

La presente revisión proporciona una mirada de la literatura reciente sobre la alienación parental, configurándose como un aporte teórico y bibliográfico sobre el tema. Se ha identificado que la alienación parental se manifiesta en una importante cantidad de personas que refieren experimentar o haberla experimentado y que además se vincula con un deterioro en la salud mental en la etapa adulta. En este sentido, se denotan vacíos en la literatura, como es la necesidad de aumentar los estudios de prevalencia del fenómeno en distintas latitudes del mundo, como también acrecentar mayores investigaciones que consideren la multidimensionalidad del fenómeno. El desarrollar investigaciones contingentes y a nivel hispanoparlante podría evidenciar cómo se expresa esta dinámica relacional en otros contextos culturales y situarla como una forma de maltrato infantil, por ende, promover su prevención y detección.

\section{Referencias}

Aloia, L. S. y Strutzenberg, C. (2019). Parent-child communication apprehension: The role of alienation and self-esteem. Communication reports, 32(1), 1-14. https://doi.org/c7g6

Antón, M. P. y Pérez, A. M. (2019). Estudio multidisciplinar sobre interferencias parentales. Dykinson.

Baker, A. (2005). The Long-term effects of parental alienation on adult children: A qualitative research study. American Journal of Family Therapy, 33(4), 289-302. https://doi.org/dxvxmt

Baker, A. (2009). Adult recall of parental alienation in a community sample: Prevalence and associations with psychological maltreatment. Journal of divorce and remarriage, 51(1), 16-35. https://doi.org/10.1080/10502550903423206

Baker, A. (2020). Reliability and validity of the four-factor model of parental alienation. Journal of family therapy, 42(1), 100-118. https://doi.org/10.1111/1467-6427.12253

Baker, A. y Verrocchio, M. C. (2016). Exposure to parental alienation and subsequent anxiety and depression in Italian adults. American journal of family therapy, 44(5), 255-271. https://doi.org/10.1080/01926187.2016.1230480 
Balmer, S., Matthewson, M. y Haines, J. (2018). Parental alienation: Targeted parent perspective Australian journal of psychology, 70(1), 91-99. https://doi.org/10.1111/ajpy.12159

Bentley, C. y Matthewson, M. (2020). The not forgotten child: alienated adult children's experience of parental alienation. American journal of family therapy, 48(5), 509-529. https://doi.org/10.1080/01926187.2020.1775531

Bernet, W., Gregory, N., Reay, K. y Rohner, R. (2018). An objective measure of splitting in parental alienation: The parental acceptance-rejection questionnaire. Journal of forensic sciences, 63(3), 776-783. https://doi.org/10.1111/1556-4029.13625

Bernet, W., Gregory, N., Rohner, R. y Reay, K. (2020). Measuring the difference between parental alienation and parental estrangement: The PARQ-Gap. Journal of forensic sciences, 65(4), 1225-1234. https://doi.org/10.1111/1556-4029.14300

Corrás, T., Seijo, D., Fariña, F., Novo, M., Arce, R. y Cabanach, R. G. (2017). What and how much do children lose in academic settings owing to parental separation? Frontiers in psychology, 8, 1545. https://doi.org/10.3389/ fpsyg.2017.01545

Demby S. L. (2016). Parenting coordination: applying clinical thinking to the management and resolution of post-divorce conflict. Journal of clinical psychology, 72(5), 458-468. https://doi.org/10.1002/jclp.22261

Duerr, H. y Hautzinger, M. (2019). Quantifying the degree of interparental conflict - the spectrum between conflict and forms of maltreatment and abuse. Child indicators research, 12(1), 319-330. https://doi.org/10.1007/s12187-018-9556-1

Gardner, R. A. (1985). Recent trends in divorce and custody litigation. The Academy forum, 29 (2), 3-7. https://tinyurl.com/2p8z9n8e

Gardner, R. A. (2002). Parental alienation syndrome vs. parental alienation: Which diagnosis should evaluators use in child-custody disputes? American journal of family therapy, 30(2), 93-115. https://doi.org/10.1080/019261802753573821

González, I. (2017). Las interferencias parentales y la alienación parental en el contexto jurídijurídico español: Revisión de sentencias judiciales en materia de guarda y custodia (Tesis de doctorado). Universitat de Valencia, Departament de Psicologia Bàsica. https://tinyurl.com/2p8s737m

Harman, J. J., Bernet, W. y Harman, J. (2019). Parental alienation: The blossoming of a field of study. Current directions in psychological science, 28(2), 212-217. https://doi.org/10.1177/0963721419827271 
Harman, J. J., Kruk, E. y Hines, D. A. (2018). Parental alienating behaviors: An unacknowledged form of family violence. Psychological bulletin, 144(12), 1275-1299. https://doi.org/10.1037/bul0000175

Harman, J.J., Leder-Elder, S. y Biringen, Z. (2016). Prevalence of parental alienation drawn from a representative poll. Children and youth services review, 66, 62-66. https://doi.org/f8t4dd

Harold, G. T., Elam, K. K., Lewis, G., Rice, F. y Thapar, A. (2012). Interparental conflict, parent psychopathology, hostile parenting, and child antisocial behavior: Examining the role of maternal versus paternal influences using a novel genetically sensitive research design. Development and psychopathology, 24(04), 1283-1295. https://doi.org/g7sw

Harold, G.T. y Sellers, R. (2018). Annual research review: Interparental conflict and youth psychopathology an evidence review and practice focused update. Journal of child psychology and psychiatry, 59(4), 374-402. https://doi.org/10.1111/jcpp.12893

Jaffe, A. M., Thakkar, M. J. y Piron, P. (2017). Denial of ambivalence as a hallmark of parental alienation. Cogent psychology, 4(1). https://doi.org/10.1080/23311908.2017.1327144

Johnston, J. y Sullivan, M. (2020). Parental alienation: In search of common ground for a more differentiated theory. Family court review, 58(2), 270-292. https://doi.org/g7s5

Kelly, J. B. y Johnston, J. R. (2001). The alienated child: A reformulation of parental alienation syndrome. Family court review, 39(3), 249-266. https://doi.org/c2vbfp

Kruk, E. (2018). Parental alienation as a form of emotional child abuse: Current state of knowledge and future directions for research. Family science review, 22(4), 141-162. https://tinyurl.com/4j72hate

Lee-Maturana, S., Matthewson, M. y Dwan, C. (2020). Targeted parents surviving parental aliealienation: Consequences of the alienation and coping strategies. Journal of child and family studies, 29(8), 2268-2280. https://doi.org/10.1007/s10826-020-01725-1

Linares, J. (2015). Prácticas alienadoras familiares. Gedisa.

Lorandos, D., Bernet, W. y Sauber, S. R. (2013). Parental alienation: The handbook for mental health and legal professionals. Charles C Thomas.

McTavish, J. R., MacGregor, J. C., Wathen, C. N. y MacMillan, H. L. (2016). Children's exposure to intimate partner violence: an overview. International review of psychiatry, 28(5), 504518. https://doi.org/10.1080/09540261.2016.1205001 
Mercer, J. (2019). Examining parental alienation treatments: Problems of principles and practices. Child and adolescent social work journal, 36(4), 351-363. https://doi.org/g7s7

Moher, D., Shamseer, L., Clarke, M., Ghersi, D., Liberati, A., Petticrew, M., Shekelle, P., Stewart, L. A. y PRISMA-P Group (2015). Preferred reporting items for systematic review and meta-analysis protocols (PRISMA-P) 2015 statement. Systematic reviews, 4(1), 1. https://doi.org/10.1186/2046-4053-4-1

O'Donohue, W., Benuto, L. T. y Bennett, N. (2016). Examining the validity of parental alienation syndrome. Journal of child custody: Research, issues, and practices, 13(2-3), 113-125. https://doi.org/10.1080/15379418.2016.1217758

Reay, K. M. (2015). Family reflections: A promising therapeutic program designed to treat severely alienated children and their family system. American journal of family therapy, 43(2), 197-207. https://doi.org/10.1080/01926187.2015.1007769

Rowlands, G. A. (2020). Parental alienation: A measurement tool confirmatory factor analy-sis validation study. Journal of divorce \& remarriage, 61(2), 127-147. https://doi.org/10.1080/10502556.2019.1627162

Shamseer, L., Moher, D., Clarke, M., Ghersi, D., Liberati, A., Petticrew, M., Shekelle, P. y Stewart, L. A. (2015). Preferred reporting items for systematic review and meta-analysis protocols (PRISMA-P) 2015: elaboration and explanation. BMJ, 349, g7647. https://doi.org/10.1136/bmj.g7647

Van der Wal, R. C., Finkenauer, C. y Visser, M. M. (2019). Reconciling mixed findings on children's adjustment following high-conflict divorce. Journal of child and family studies, 28(2), 468-478. https://doi.org/10.1007/ s10826-018-1277-z

Verrocchio, M. C., Baker, A. J. L. y Marchetti, D. (2018). Adult report of childhood exposure to parental alienation at different developmental time periods. Journal of family therapy, 40(4), 602-618. https://doi.org/10.1111/1467-6427.12192

Verrocchio, M. C., Marchetti, D., Carrozzino, D., Compare, A. y Fulcheri, M. (2019). Depression and quality of life in adults perceiving exposure to parental alienation behaviors. Health and quality of life outcomes, 17(1), 14. https://doi.org/10.1186/s12955-019-1080-6

Vilalta, R. y Winberg, M. (2017). On the myth of parental alienation syndrome (PAS) and the DSM-5. Papeles del psicólogo, 38(3), 224-231. https://doi.org/g7tb 
Visser, M., Finkenauer, C., Schoemaker, K., Kluwer, E., van der Rijken, R., van Lawick, J., Bom, H., de Schipper, J. C. y Lamers-Winkelman, F. (2017). I'll never forgive you: High conflict divorce, social network, and co-parenting conflicts. Journal of child and family studies, 26(11), 3055-3066. https://doi.org/10.1007/s10826-017-0821-6

Warshak, R. A. (2020). When evaluators get it wrong: False positive IDs and parental alienation Psychology, public policy, and law, 26(1), 54 -68. https://doi.org/10.1037/law0000216

\section{Para citar en APA}

Portilla-Saavedra, D., Moya Vergara, R. \& Pinto-Cortez, C. (2021). Perspectivas y tendencias actuales del concepto de alienación parental: una revisión sistemática de la literatura. Terapia Psicológica (En línea), 39(2), 237-255. https://doi.org/10.4067/S0718-48082021000200237 